\title{
High-Yield Production of Monolayer FePS 3 Quantum Sheets via Chemical Exfoliation for Efficient Photocatalytic Hydrogen Evolution
}

\author{
Zhongzhou Cheng, Tofik Ahmed Shifa, Fengmei Wang, Yi Gao, Peng He, Kai Zhang,* \\ Chao Jiang, Quanlin Liu, and Jun $\mathrm{He}^{* *}$
}

2D layered transition metal phosphorus trichalcogenides $\left(\mathrm{MPX}_{3}\right)$ possess higher in-plane stiffness and lower cleavage energies than graphite. This allows them to be exfoliated down to the atomic thickness. However, a rational exfoliation route has to be sought to achieve surface-active and uniform individual layers. Herein, monolayered $\mathrm{FePS}_{3}$ quantum sheets (QSs) are systematically obtained, whose diameters range from 4-8 nm, through exfoliation of the bulk in hydrazine solution. These QSs exhibit a widened bandgap of $2.18 \mathrm{eV}$ as compared to the bulk $(1.60 \mathrm{eV}) \mathrm{FePS}_{3}$. Benefitting from the monolayer feature, $\mathrm{FePS}_{3}$ QSs demonstrate a substantially accelerated photocatalytic $\mathrm{H}_{2}$ generation rate, which is up to three times higher than the bulk counterpart. This study presents a facile way, for the first time, of producing uniform monolayer $\mathrm{FePS}_{3}$ QSs and opens up new avenues for designing other low-dimensional materials based on $\mathrm{MPX}_{3}$.

monolayer and found a remarkably high value of $625.9 \mathrm{~cm}^{2} \mathrm{~V}^{-1} \mathrm{~s}^{-1}$ at room temperature, ${ }^{[9]}$ which is even higher than other typical 2D materials such as monolayer $\mathrm{MoS}_{2}\left(200 \mathrm{~cm}^{2} \mathrm{~V}^{-1} \mathrm{~s}^{-1}\right)^{[10]}$ and $\mathrm{WS}_{2}$ $\left(214 \mathrm{~cm}^{2} \mathrm{~V}^{-1} \mathrm{~s}^{-1}\right)$. Although it is still under development, catalytic applications of nanostructured materials in this family have notable promising features. Theoretical speculations ${ }^{[9]}$ reveal that the positions of their conduction and valance bands energy levels straddle the water redox potentials, making them appealing for photocatalyzing water splitting reactions. Moreover, the calculated high carrier mobilities of the $2 \mathrm{D} \mathrm{MPX}_{3}$ materials such as $\mathrm{MnPSe}_{3}$ indicate that the transfer of carriers to reactive sites would be easier

Owing to their interesting and useful properties, 2D materials, such as graphene, ${ }^{[1]} \mathrm{MoS}_{2},{ }^{[2]}$ and g- ${ }_{3} \mathrm{~N}_{4},{ }^{[3]}$ have led to the increasing attention of scientific communities during the last decades. The size effects exhibited by few-layered nanosheets of these materials result in fundamentally unique properties that substantially differ from their bulk counterparts. ${ }^{[4]}$ Nowadays, a new class of 2D layered metal phosphorus trichalcogenides $\left(\mathrm{MPX}_{3}\right.$, where $\mathrm{M}=\mathrm{Fe}, \mathrm{Mn}$, Ni, etc. and $\mathrm{X}=\mathrm{S}$ or Se) have received tremendous attentions. ${ }^{[5-7]}$ It is gratifying that various important findings have been reported regarding the $\mathrm{MPX}_{3}$ materials in different applications. ${ }^{[8]}$ For instance, Zhang et el., have recently calculated the carrier mobility of $2 \mathrm{D} \mathrm{MnPSe}_{3}$ in the photocatalytic process, reducing the possibility of electron-hole recombination. It is also important to note that the different kinds of elements in $\mathrm{MPX}_{3}$ (particularly the calchogen and the metal atom) give rise to the variation of the bandgaps from 1.3 to $3.5 \mathrm{eV}^{[5]}$ This rich and appropriate bandgaps bring the possibility to efficiently use visible light and design best performing photocatalysts. Our group has recently reported a systematic way of synthesizing ultrathin $2 \mathrm{D} \mathrm{NiPS}{ }_{3}^{[11]}$ and $\mathrm{MnPX}_{3}(\mathrm{X}=\mathrm{S} \text { and } \mathrm{Se})^{[12]}$ nanosheets. These experimental realizations communicated the promising hydrogen evolving activities of $\mathrm{MPX}_{3}$ under illumination of simulated solar light without cocatalyst or sacrificial agents. In line with this, the

Z. Z. Cheng, T. A. Shifa, Dr. F. M. Wang, P. He, Prof. C. Jiang, Prof. J. He CAS Center for Excellence in Nanoscience

CAS Key Laboratory of Nanosystem and Hierarchical Fabrication

National Center for Nanoscience and Technology

Beijing 100190, China

E-mail: hej@nanoctr.cn

Z. Z. Cheng, Prof. Q. L. Liu

School of Materials Science and Engineering

University of Science and Technology

Beijing 100083, China

T. A. Shifa, Prof. J. He

University of Chinese Academy of Sciences

Beijing 100049, China

\author{
Y. Gao, Prof. K. Zhang \\ i-Lab \\ Suzhou Institute of Nano-Tech and Nano-Bionics \\ Chinese Academy of Sciences \\ Suzhou 215123, Jiangsu, P. R. China \\ E-mail: kzhang2015@sinano.ac.cn \\ Y. Gao \\ Hubei Collaborative Innovation Center for Advanced Organic Chemical \\ Materials-Hubei Key Laboratory of Ferro and Piezoelectric Materials \\ and Devices \\ Faculty of Physics and Electronic Sciences \\ Hubei University \\ Wuhan 430062, Hubei, P. R. China
}

The ORCID identification number(s) for the author(s) of this article can be found under https://doi.org/10.1002/adma.201707433.

DOI: 10.1002/adma.201707433 
issue of performance enhancement in photocatalysis is associated with strong light harvesting capability in the visible and near-infrared region, multiple charge carrier generation, and large surface area to volume ratio. Quantum sheets (QSs) are the best candidates to virtually meet these requirements. ${ }^{[13,14]}$ Recent studies show that the QSs demonstrate a particularly prominent multiple-exciton generation effect, which is considered as a promising way to reduce heat-related energy losses in solar system by splitting one high-energy photon into multiple low-energy excitons thereby increasing energy conversion efficiency. ${ }^{[15,16]}$ Given the very promising features of $\mathrm{MPX}_{3}$ in catalysis and the attributes of QSs in photochemistry, the design of quantum confined materials in this family is of a great benefit to realize multiply advantageous photocatalyst.

Here, we made use of an important concept to enable us design QSs. That is, most of the $\mathrm{MPX}_{3}$ compounds have higher in-plane stiffness $\left(60-120 \mathrm{~N} \mathrm{~m}^{-1}\right)$ and lower cleavage energies $\left(0.29-0.54 \mathrm{~J} \mathrm{~m}^{-2}\right)^{[9]}$ as compared to graphite $\left(0.37 \mathrm{~J} \mathrm{~m}^{-2}\right) \cdot{ }^{[17]}$ This suggests that the surface-active $\mathrm{MPX}_{3}$ nanosheet can be practically exfoliated from the bulk. ${ }^{[18,19]}$ Following this, we employ a facile way to exfoliate layered $\mathrm{FePS}_{3}$ into monolayer QSs with a uniform lateral size of 4-8 $\mathrm{nm}$. Benefitting from our facile exfoliation, the in-plane surface is greatly altered toward exposing more active sites, which is prominent feature of efficient catalysis. Accordingly, photocatalytic test shows that the hydrogen generation rate of the $\mathrm{FePS}_{3} \mathrm{QSs}$ is up to three times $\left(290 \mu \mathrm{mol} \mathrm{g}^{-1} \mathrm{~h}^{-1}\right)$ higher than that of bulk FePS

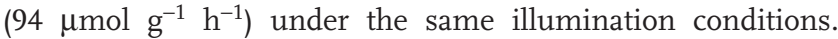

We believe that, this work opens up a clear avenue for investigation of other members in this family at the level of QSs.

The bulk $\mathrm{FePS}_{3}$ crystal was synthesized by chemical vapor transport (CVT) method in a sealed quartz tube as can be seen from the schematic diagram in Figure 1a. The scanning electron microscopy (SEM) image of the as prepared hexagonal $\mathrm{FePS}_{3}$ sheet, with a size about $200 \mu \mathrm{m}$, and the corresponding energy dispersive X-ray spectroscopy (EDS) elemental mapping images are clearly shown in Figure 1b. More crystal structures of the bulk FePS 3 are displayed in Figures S1 and S2 in the Supporting Information. To obtain the desired FePS $\mathrm{QSS}_{3}$, a two-step exfoliation method was implemented. First, the prepared bulk $\mathrm{FePS}_{3}$ was intercalated by reacting with hydrazine $\left(\mathrm{N}_{2} \mathrm{H}_{4}\right)$ in hydrothermal condition (Figure 1c). Second, the intercalated $\mathrm{FePS}_{3}$ crystals were exfoliated by sonication at a high power of $100 \mathrm{~W}$ for $4 \mathrm{~h}$ to form the QSs, as shown in Figure 1d. It can be seen that the QSs are evenly distributed on the substrate. The statistical distribution of the diameter (inset) illustrates that the isolated QSs tend to be $4-8 \mathrm{~nm}$ in size. This may provide better homogeneity for further characterization and testing. More transmission electron microscopy (TEM) images of the QSs are depicted in Figure S3 of the Supporting Information. It is obvious that the $\mathrm{FePS}_{3} \mathrm{QSs}$, with the size below $10 \mathrm{~nm}$, are well dispersed on the ultrathin carbon film with a homogeneous thickness (Figure S3b,c, Supporting Information). The mechanism of the exfoliation processes can reasonably be explained by a redox rearrangement model. ${ }^{[20]}$ At the first glance, part of the $\mathrm{N}_{2} \mathrm{H}_{4}$ were oxidized to $\mathrm{N}_{2} \mathrm{H}_{5}{ }^{+}$
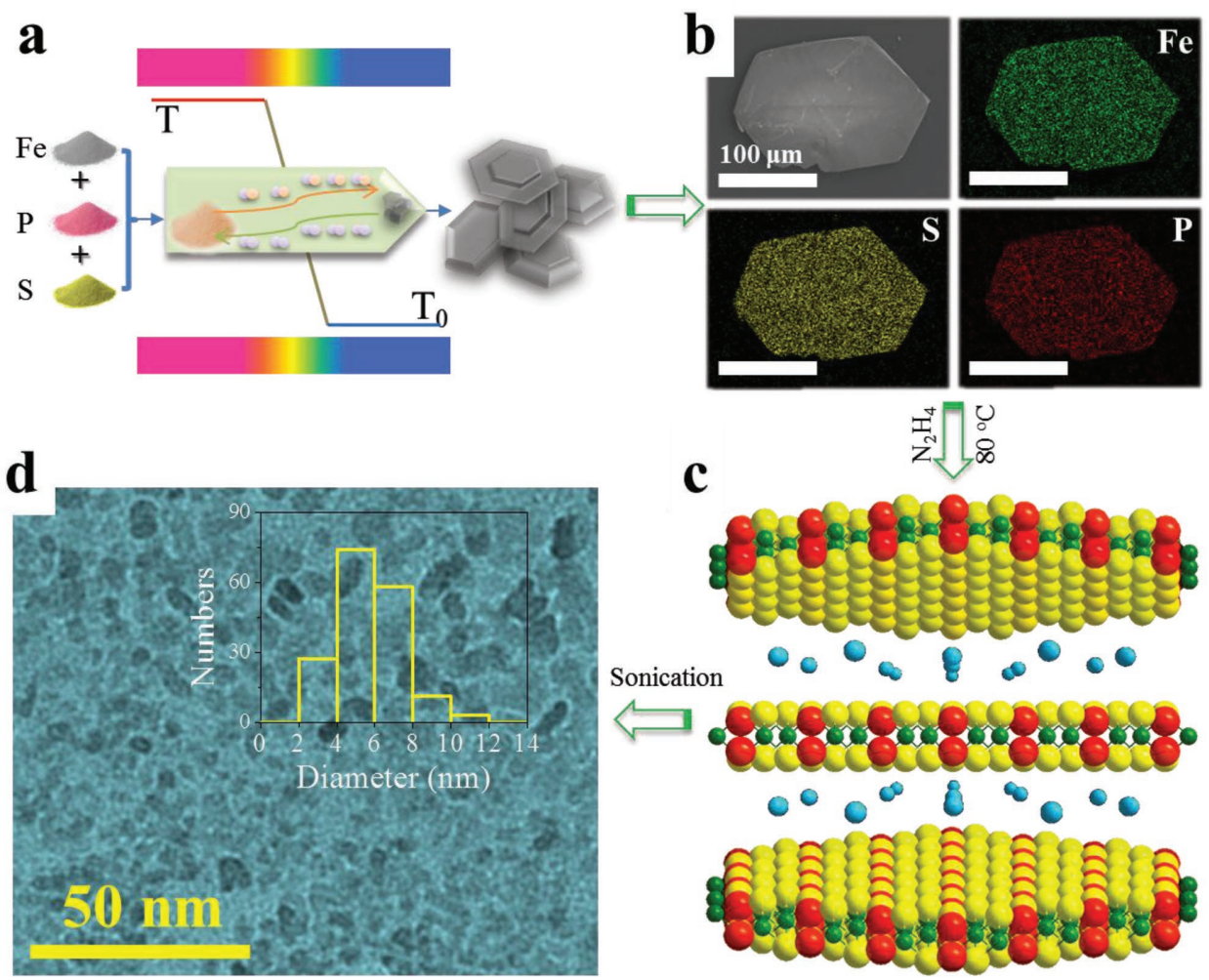

Figure 1. Preparation of the $\mathrm{FePS}_{3}$ bulk and QSs. a) The schematic of chemical vapor transport (CVT) method. b) SEM and the corresponding EDS elemental mapping images of a bulk $\mathrm{FePS}_{3}$ as prepared. c) The decomposition product of pre-exfoliated bulk FePS $(\mathrm{Fe}$ : green, P: red, S: yellow). d) The TEM image of the $\mathrm{FePS}_{3}$ QSs. A diameter statistic of the size distribution of FePS $\mathrm{QSS}_{3}$ is given in the inset. 
during intercalation. Up on heating, the intercalated $\mathrm{N}_{2} \mathrm{H}_{5}{ }^{+}$was decomposed to different gaseous species such as $\mathrm{N}_{2}, \mathrm{NH}_{3}$, and $\mathrm{H}_{2}$ owing to its poor thermal stability. This brought the formation of expanded crystals in $\mathrm{FePS}_{3}$, which after were treated at high power sonication. The ultrasonic waves in solvent would generate cavitation bubbles and then collapse into high-energy jets, breaking up the expanded crystals and producing QSs. In addition, modeling has shown that if the surface energy of the solvent is similar to that of the layered material, the energy difference between the exfoliated and reaggregated states will be very small, removing the driving force for reaggregation. ${ }^{[21-23]}$ For example, graphene, h-BN, transition metal dichalcogenides materials, and some transition metal oxide materials have been exfoliated by using suitable solvents such as $N$-methyl-pyrrolidone. ${ }^{[24]}$ In our system, $\mathrm{FePS}_{3}$ material was estimated to have a surface energy of $\approx 100 \mathrm{~mJ} \mathrm{~m}{ }^{-2} \cdot{ }^{[9,25]}$ On the other hand, the solvent surface energy is related to the surface tension by ${ }^{[23]}$

$E_{\text {sur }}^{\text {sol }}=\gamma+T S_{\text {sur }}^{\text {sol }}$

where $\gamma$ is the solvent surface tension $\left(\mathrm{mN} \mathrm{m}^{-1}\right), E_{\text {sur }}^{\text {sol }}$ is the solvent surface energy $\left(\mathrm{mJ} \mathrm{m}^{-2}\right), T$ is the temperature $(\approx 300 \mathrm{~K})$, and $S_{\text {sur }}^{\text {sol }}$ is the solvent surface entropy $\left(\approx 0.1 \mathrm{~mJ} \mathrm{~m}^{-2} \mathrm{~K}^{-1}\right)$. Given the surface tension of water $\left(\gamma=72 \mathrm{mN} \mathrm{m}^{-1}\right.$ at $\left.300 \mathrm{~K}\right)$ and hydrazine hydrate $\left(\gamma=74 \mathrm{mN} \mathrm{m}^{-1}\right.$ at $\left.300 \mathrm{~K}\right)$, their surface energy can be calculated as 102 and $104 \mathrm{~mJ} \mathrm{~m}^{-2}$, respectively, which are very close to that of $\mathrm{FePS}_{3}$. This result means that water and hyadrazine hydrate are suitable solvents to realize the successful exfoliation into monolayer $\mathrm{FePS}_{3} \mathrm{QSs}$.

In order to have a detailed examination of the samples, we characterized the obtained materials by high-resolution transmission electron microscopy (HRTEM). The HRTEM image of the bulk FePS 3 was shown Figure 2a. The space of $0.321 \mathrm{~nm}$ can be indexed to the (002) plane of $\mathrm{FePS}_{3}$. A clear observation is apparent from Figure $2 \mathrm{~b}$ from which $\mathrm{a} \approx 7 \mathrm{~nm}$ diameter of quantum sheet (QS) can be seen. Furthermore, the lattice stripes with space of $0.186 \mathrm{~nm}$ belonging to the (310) plane of $\mathrm{FePS}_{3}$ are observed, solidly corroborating the formation of the desired material. More details about the crystal structure of the bulk and QSs are displayed in Figures S3-S5 in the Supporting Information. For visualization through atomic force microscopy (AFM), the FePS ${ }_{3}$ QSs were drop-casted onto sapphire, by a $20 \mu \mathrm{L}$ pipette. The thickness profile of the QSs reveals that most of the QSs are monolayers (Figure S6, Supporting Information).

To further confirm the crystallographic assertion, the X-ray diffraction (XRD) patterns of the $\mathrm{FePS}_{3}$ bulk and QSs were obtained and displayed in Figure 2c. Besides the peaks belonging to fluorine-doped tin oxide (FTO) substrate (The International Centre for Diffraction Data $\langle$ ICDD $\rangle$, PDF\#46-1088), a perfect alignment with the standard card (ICDD, PDF\#30-0663) for all the peaks of bulk FePS 3 suggests the high phase purity of our synthesized material. The sharp peaks at $13.8^{\circ}$ and $27.8^{\circ}$ can be indexed to (001) and (002) planes of the $\mathrm{FePS}_{3}$ crystals, respectively. Comparing the QSs with the bulk, it is obvious that the peak at $13.8^{\circ}$ is disappeared
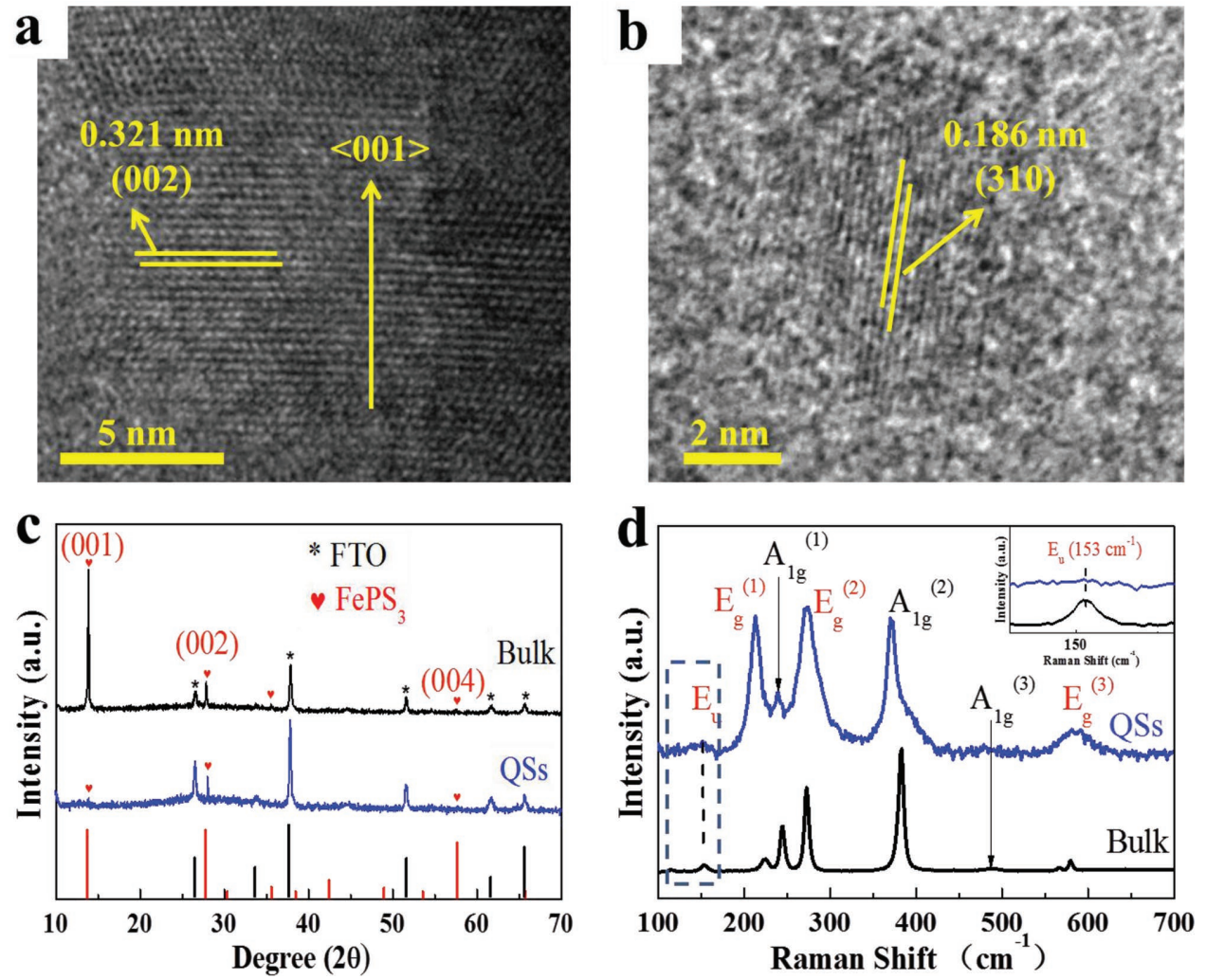

Figure 2. Characterization of the FePS 3 bulk and QSs. HRTEM of: a) the FePS 3 bulk and b) QSs. c) XRD and d) Raman spectra of the bulk and QSs samples. 
for the case of QSs. This can be attributed to the diminishing of (001) plane as the bulk FePS $\mathrm{S}_{3}$ crystals is transformed into monolayer. We also characterized the surface chemical states by X-ray photoelectron spectroscopy (XPS), shown in Figure S7 in the Supporting Information. Accordingly, the high-resolution XPS spectra of $\mathrm{Fe} 2 \mathrm{p}$ region indicate that the oxidation state of $\mathrm{Fe}$ in $\mathrm{FePS}_{3}$ is +2 . A critical comparison of XPS spectra (bulk vs QSs) reveals that there is no obvious change on the surface chemical states, further corroborating the safety and stability of our exfoliation method.

The analysis of Raman spectra gives more insight understanding of the distinction between the bulk and monolayer QSs, displayed in Figure 2d. According to the report by Scagliotti et al., ${ }^{[26]}$ the Raman modes of $\mathrm{FePS}_{3}$ originate from two parts of the crystal structure (the vibrations of metal atom and the $P_{2} S_{6}$ unit, which belongs to the $D_{3 \mathrm{~d}}$ symmetry group). Three $\mathrm{A}_{1 \mathrm{~g}}$-type modes (polarized, $\mathrm{A}_{1 \mathrm{~g}}{ }^{(1-3)}$ ) and three $\mathrm{E}_{\mathrm{g}}$-type modes (depolarized, $\mathrm{E}_{\mathrm{g}}{ }^{(1-3)}$ ) from the vibrations of the $P_{2} S_{6}$ unit could be resolved in the spectra (Figure S8, Supporting Information). As shown in Figure $2 \mathrm{~d}$, the peaks at $214 \mathrm{~cm}^{-1}\left(\mathrm{E}_{\mathrm{g}}{ }^{(1)}\right), 240 \mathrm{~cm}^{-1}$ $\left(\mathrm{A}_{1 \mathrm{~g}}{ }^{(1)}\right), 274 \mathrm{~cm}^{-1}\left(\mathrm{E}_{\mathrm{g}}{ }^{(2)}\right), 372 \mathrm{~cm}^{-1}\left(\mathrm{~A}_{1 \mathrm{~g}}{ }^{(2)}\right)$, and $586 \mathrm{~cm}^{-1}\left(\mathrm{E}_{\mathrm{g}}{ }^{(3)}\right)$ can be assigned to the FePS 3 QSs, and those at $153 \mathrm{~cm}^{-1}\left(\mathrm{E}_{\mathrm{u}}\right)$, $223 \mathrm{~cm}^{-1}\left(\mathrm{E}_{\mathrm{g}}{ }^{(1)}\right), 245 \mathrm{~cm}^{-1}\left(\mathrm{~A}_{1 \mathrm{~g}}{ }^{(1)}\right), 272 \mathrm{~cm}^{-1}\left(\mathrm{E}_{\mathrm{g}}{ }^{(2)}\right)$, and $382 \mathrm{~cm}^{-1}$ $\left(\mathrm{A}_{1 \mathrm{~g}}{ }^{(2)}\right), 486 \mathrm{~cm}^{-1}\left(\mathrm{~A}_{1 \mathrm{~g}}{ }^{(3)}\right)$, and $\approx 580 \mathrm{~cm}^{-1}\left(\mathrm{E}_{\mathrm{g}}{ }^{(3)}\right)$ can be associated to the bulk FePS $3 .{ }^{[26-28]}$ The $\mathrm{A}_{1 \mathrm{~g}}$ modes represent the stretching vibration of the $P-P$ band, which indicate the out-of plane vibrations of the $P_{2} S_{6}$ unit (Figure $\mathrm{S} 8$, Supporting Information). The $\mathrm{A}_{1 \mathrm{~g}}{ }^{(1)}$ is due to the opposite movement of the $S_{3} P-P S_{3}$ unit, which has been found very sensitive to alkali-ion intercalation and to the $c$-axis expansion. Moreover, the $\mathrm{A}_{1 \mathrm{~g}}{ }^{(2)}$ and $\mathrm{A}_{1 \mathrm{~g}}{ }^{(3)}$ modes represent the symmetric stretching vibration of the $P-S$ bonds and the relative movement of the $S_{3} P-P S_{3}$ unit, respectively. Moreover, the $E_{g}$ modes are meant for the tangential vibration of the $P-P$ bond, which indicate the in-plane vibrations of the $P_{2} S_{6}$ unit (Figure S8, Supporting Information). The $E_{g}{ }^{(1)}$ and $E_{g}{ }^{(2)}$ are active in two orthogonal scattering geometries where as the $\mathrm{E}_{\mathrm{g}}{ }^{(3)}$ is sensitive to the lattice distortions. ${ }^{[26]}$ The peak at about $153 \mathrm{~cm}^{-1}$ is the Raman counterpart of the strongly infrared active $\mathrm{E}_{\mathrm{u}}$-type mode of the $P_{2} S_{6}$ unit observed in all members of the $\mathrm{MPS}_{3}$ compound absorption spectra.
When the unit cell is doubled along the $\mathrm{c}$ axis, the out-ofplane vibration of two $P_{2} X_{6}$ units in adjacent layers becomes Raman active. ${ }^{[26,29]}$ From the inset of Figure 2d, the absence of $\mathrm{E}_{\mathrm{u}}$ peak in QSs sample suggests the achievement of monolayer $\mathrm{FePS}_{3}$ QSs.

To explore the optical properties of the FePS bulk and QSs, UV/vis/NIR diffuse-reflection spectra (DRS) and ultraviolet photoelectron spectroscopy (UPS) were used and the results are shown in Figure 3. According to the Kubelka-Munk theory, the absorption coefficient $(\alpha)$ could be obtained by the KubelkaMunk function $(F(R))$ from the diffuse reflectance measurement. ${ }^{[30]}$ Also, the bandgap energy of the material is estimated by the intercept of the tangent to the $X$-axis $(h v)$ in the Tauc plot $^{[31]}$ (inset of Figure 3a). The FePS 3 is known to be an indirect bandgap semiconductor so that the $(\alpha h v)^{1 / 2}$ instead of $(\alpha h v)^{2}$ is set as the Y-axis fro contracting the Tauc plot. ${ }^{[32]}$ Accordingly, the bandgap values of $\mathrm{FePS}_{3}$ bulk and QSs are found to be 1.60 and $2.18 \mathrm{eV}$, respectively (Figure 3a, inset). The UPS was used to determine the valence band energy level $\left(\mathrm{E}_{\mathrm{v}}\right)$ of the bulk and QSs, as shown in Figure 3b. Through subtracting the width of He I UPS spectra from the excitation energy $(21.22 \mathrm{eV})$, the $E_{\mathrm{v}}$ values were calculated to be $-5.40 \mathrm{eV}$ for the bulk and $-5.57 \mathrm{eV}$ for the QSs (vs $E_{\text {Vacuum }}$ ), respectively. Then the conduction band energy $\left(E_{c}\right)$ of the material can be calculated by $E_{v}-E_{g}$ $(-3.80 \mathrm{eV}$ for bulk and $-3.39 \mathrm{eV}$ for QSs). As a matter of fact, QSs exhibit a wider bandgap $(2.18 \mathrm{eV})$, compared to the bulk $(1.60 \mathrm{eV})$. No matter how the $\mathrm{FePS}_{3} \mathrm{QSs}$ display a more negative overpotential than $\mathrm{H}^{+} / \mathrm{H}_{2}(-0.20 \mathrm{~V}$ for bulk and $-0.61 \mathrm{~V}$ for QSs, vs NHE, PH = 7), the feature of QSs enables the accumulation of abundant hot electrons at $\mathrm{E}_{\mathrm{c}}$ for catalyzing the hydrogen evolution reaction (Figure S9, Supporting Information).

Having seen the well-exfoliated QSs of $\mathrm{FePS}_{3}$ and the appropriately positioned $\mathrm{E}_{\mathrm{c}}$ level for sunlight driven catalysis of $\mathrm{H}_{2}$ gas production, we conducted photocatalytic hydrogen evolution experiments in aqueous solution containing $10 \mathrm{vol} \%$ triethanolamine (TEOA) as the hole scavenger. The performance of bulk $\mathrm{FePS}_{3}$ was also measured under exactly the same condition for the sake of comparison. As can be seen in Figure 4a, $\mathrm{FePS}_{3}$ QSs show a high efficiency of $290 \mu \mathrm{mol} \mathrm{h} \mathrm{h}^{-1} \mathrm{~g}^{-1} \mathrm{com}$ pared with the significantly lower value of $94 \mu \mathrm{mol} \mathrm{h}^{-1} \mathrm{~g}^{-1}$ for
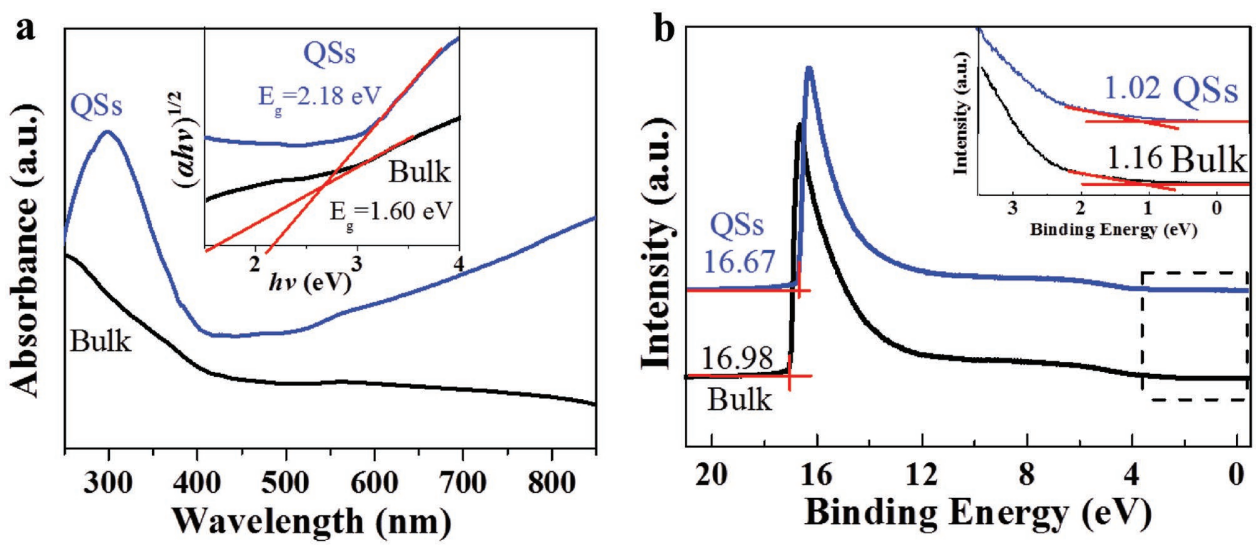

Figure 3. Optical properties of the $\mathrm{FePS}_{3}$ bulk and QSs. a) UV/vis/NIR diffusive reflectance absorption spectra and estimated bandgap potential (inset) of synthesized FePS 3 bulk $(1.60 \mathrm{eV})$ and QSs $(2.18 \mathrm{eV})$. b) UPS spectra of the bulk and QSs, in which the dashed red lines mark the baseline and the tangents of the curve. 

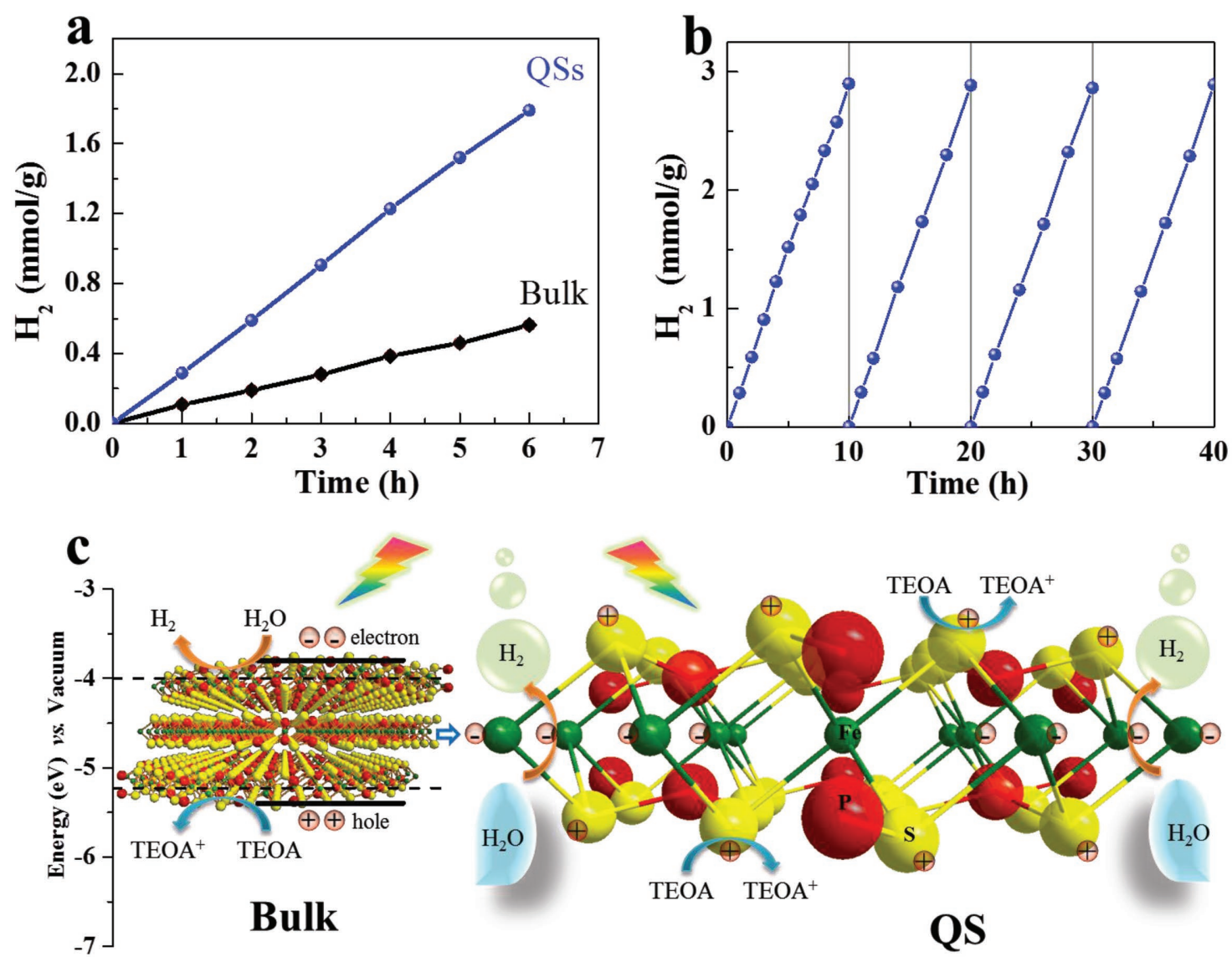

Figure 4. photocatalytic performance of the FePS $S_{3}$ bulk and QSs. a) Plots of hydrogen production through direct photocatalytic water splitting of the $\mathrm{FePS}_{3}$ bulk and QSs, in $100 \mathrm{~mL}$ deionized water with $10 \%$ TEOA as sacrificial agent. b) Cycling measurements of hydrogen gas generation of the FePS QSs. c) Schematics of photocatalytic reaction processes for the FePS 3 bulk and QSs.

bulk FePS 3 . Furthermore, the stability of QSs for hydrogen production was evaluated and the result is depicted in Figure 4b. The sample exhibits a feasible ability in the cycling measurements of $\mathrm{H}_{2}$ generation under light illumination for a continuous $40 \mathrm{~h}$ test. This enhancement can be attributed to the great amount of exposed boundaries providing more active sites and efficient separation of photogenerated electrons in QSs. From such all dimensional quantum confinement, it can be inferred that there are significant differences from the bulk in photocatalytic reaction, as shown in Figure 4c. Under the light illumination, the photogenerated electron and hole are located in the conduction band and valence band separately. The photogenerated electrons are consumed to produce $\mathrm{H}_{2}$ molecules while the photogenerated holes are used to oxide the sacrificial agent (TEOA). Exfoliating the bulk into monolayered $\mathrm{FePS}_{3}$ QSs highly contributes to the increase in surface area/active sites, which is beneficial for the enhancement of photocatalytic activity.

In summary, we have developed a facile way to obtain monolayer $\mathrm{FePS}_{3} \mathrm{QSs}$ from the bulk $\mathrm{FePS}_{3}$, which was first prepared by CVT method. The QSs demonstrate a lateral size below $10 \mathrm{~nm}$. The exfoliation method brought no change on the chemical composition and state of the elements in $\mathrm{FePS}_{3}$. Given the 3D confinement, there resulted an associated change in the electronic band structure of the obtained QSs as compared to the bulk. A three times performance enhancement (290 $\mu \mathrm{mol} \mathrm{h} \mathrm{h}^{-1} \mathrm{~g}^{-1}$ for the QSs and $94 \mu \mathrm{mol} \mathrm{h} \mathrm{h}^{-1} \mathrm{~g}^{-1}$ for bulk) is observed for QSs in photocatalytic hydrogen evolution reaction that can be attributed to the monolayer effects. We believe that these facilely synthesized monolayer QSs can also find a very promising performance in other applications as well.

\section{Experimental Section}

Preparation of $\mathrm{FePS}_{3}$ Powders: A stoichiometric amount of high-purity elements (Fe: $99.99 \%$, P: $99.999 \%$, S: $99.99 \%$,) and iodine $(30 \mathrm{mg}$ ) as a transport agent were sealed into a quartz tube under vacuum and heated at $700{ }^{\circ} \mathrm{C}$ for two weeks. After cooled down to room temperature, a product of black powder with metallic luster was obtained.

Preparation of $\mathrm{FePS}_{3}$ QSs: The as-synthesized bulk FePS3 $(30 \mathrm{mg})$ with $20 \mathrm{~mL}$ of hydrazine hydrate $(80 \%)$ was sealed in an autoclave and heated at $80^{\circ} \mathrm{C}$ for $4 \mathrm{~h}$. The mixture was then sonicated in a high-power sonic bath $(100 \mathrm{~W})$ for $4 \mathrm{~h}$ to form a homogeneous suspension. Eventually, the desired $\mathrm{FePS}_{3}$ QSs were obtained by a centrifugal separation at $8000 \mathrm{rpm}$ for $15 \mathrm{~min}$ to remove unexfoliated material. For the further measurements, the QSs were spin-coated on the FTO and dried at $60{ }^{\circ} \mathrm{C}$ for $24 \mathrm{~h}$.

Characterization of Materials: SEM and the corresponding EDS mapping images were obtained from a Hitachi $\mathbf{S} 4800$ field-emission scanning electron microanalyzer with EDS. TEM and HRTEM images were obtained via employing a Tecnai G2 F20 with beam energy of $200 \mathrm{keV}$. The thickness was analyzed by AFM (MFP-3D Infinity). XRD 
patterns were collected from a D/MAX-TTRIII(CBO) system using a Cu-K $\alpha$ radiation $(\lambda=1.5418 \AA$ ). Raman spectroscopy was measured at room temperature on an inVia Renishaw system at the excitation line of $532 \mathrm{~nm}$. The Raman peak of Si at $520 \mathrm{~cm}^{-1}$ was used as a reference to calibrate the spectrometer. XPS was performed on the Thermo Scientific ESCALab 250Xi using $200 \mathrm{~W}$ monochromatic Al Ko radiation. The $500 \mu \mathrm{m} X$-ray spot was used for SAXPS analysis. The base pressure in the analysis chamber was about $3 \times 10^{-9}$ mbar. Typically, the hydrocarbon $\mathrm{Cls}$ line at $284.8 \mathrm{eV}$ from adventitious carbon is used for energy referencing. UV/vis/NIR DRS were recorded on a Lambda 750 spectrophotometer equipped with an integrating sphere. The valence band energy of the samples was analyzed on Thermo Scientific ESCALab 250Xi using UPS. The gas discharge lamp was used for UPS, with helium gas admitted and the Hel $(21.22 \mathrm{eV})$ emission line employed. The helium pressure in the analysis chamber during analysis was about $2 \times 10^{-8} \mathrm{mbar}$. The data were acquired with $-10 \mathrm{~V}$ bias.

Measurement of Photocatalytic $\mathrm{H}_{2}$ Evolution: Photocatalytic water splitting experiments were conducted in a $500 \mathrm{~mL}$ cylinder quartz reactor at ambient temperature. A $300 \mathrm{~W}$ xenon lamp used as a light source. In a typical $\mathrm{H}_{2}$ evolution experiment, the prepared photocatalyst (on FTO, about $0.6 \mathrm{mg} \mathrm{cm}$ ) was placed at the bottom of reactor containing $100 \mathrm{~mL}$ of aqueous solution with 10\% TEOA. Before irradiation, the system was vacuumed for about $30 \mathrm{~min}$ to remove the air inside and to ensure that the system was under the anaerobic condition. A certain amount of gas was intermittently sampled and analyzed by gas chromatography (GC7900, Shimadzu, Japan, TCD, nitrogen as a carrier gas and $5 \AA$ molecular sieve column) by using $\mathrm{Ar}$ as a carrier gas. A baseline was recorded for each test before exposure to xenon lamp.

\section{Supporting Information}

Supporting Information is available from the Wiley Online Library or from the author.

\section{Acknowledgements}

This work was supported by the Ministry of Science and Technology of China (No. 2016YFA0200700), the National Natural Science Foundation of China (Nos. 21703047, 21373065, 61474033, 61574050, and 11674072), Strategic Priority Research Program of the Chinese Academy of Sciences (Grant No. XDA09040201), and CAS Key Laboratory of Nanosystem and Hierarchical Fabrication. The authors also gratefully acknowledge the support of Youth Innovation Promotion Association CAS.

\section{Conflict of Interest}

The authors declare no conflict of interest.

\section{Keywords}

chemical exfoliation, $\mathrm{FePS}_{3}$, monolayer quantum sheets, water splitting

Received: December 20, 2017 Revised: March 15, 2018

Published online:
[1] K. S. Novoselov, A. K. Geim, S. V. Morozov, D. Jiang, Y. Zhang, S. V. Dubonos, I. V. Grigorieva, A. A. Firsov, Science 2004, 306, 666.

[2] B. Radisavljevic, A. Radenovic, J. Brivio, i. V. Giacometti, A. Kis, Nat. Nanotechnol. 2011, 6, 147.

[3] X. Wang, K. Maeda, A. Thomas, K. Takanabe, G. Xin, J. M. Carlsson, K. Domen, M. Antonietti, Nat. Mater. 2009, 8, 76.

[4] K. F. Mak, C. Lee, J. Hone, J. Shan, T. F. Heinz, Phys. Rev. Lett. 2010, 105, 136805

[5] B. L. Chittari, Y. Park, D. Lee, M. Han, A. H. MacDonald, E. Hwang, J. Jung, Phys. Rev. B 2016, 94, 184428.

[6] A. Wildes, V. Simonet, E. Ressouche, G. J. Mcintyre, M. Avdeev, E. Suard, S. Kimber, D. Lançon, G. Pepe, B. Moubaraki, Phys. Rev. B 2015, 92, 224408.

[7] J.-U. Lee, S. Lee, J. H. Ryoo, S. Kang, T. Y. Kim, P. Kim, C.-H. Park, J. G. Park, H. Cheong, Nano Lett. 2016, 16, 7433.

[8] M. A. Susner, M. Chyasnavichyus, M. A. McGuire, P. Ganesh, P. Maksymovych, Adv. Mater. 2017, 29, 1602852.

[9] X. Zhang, X. Zhao, D. Wu, Y. Jing, Z. Zhou, Adv. Sci. 2016, 3, 1600062.

[10] Y. Cai, G. Zhang, Y. W. Zhang, J. Am. Chem. Soc. 2014, 136, 6269.

[11] F. Wang, T. A. Shifa, P. He, Z. Cheng, J. Chu, Y. Liu, Z. Wang, F. Wang, Y. Wen, L. Liang, Nano Energy 2017, 40, 673.

[12] T. A. Shifa, F. M. Wang, Z. Z. Cheng, P. He, Y. Liu, C. Jiang, Z. Wang, J. He, Adv. Funct. Mater. 2018, 28, 1800548.

[13] X. Y. Liu, H. Chen, R. Wang, Y. Shang, Q. Zhang, W. Li, G. Zhang, J. Su, C. T. Dinh, F. de Arquer, Adv. Mater. 2017, 29, 1605646.

[14] S. Zhou, N. Liu, J. Zhao, Comput. Mater. Sci. 2017, 130, 56.

[15] M. Zamkov, Nat. Energy 2017, 2, 17072.

[16] J. B. Sambur, T. Novet, B. Parkinson, Science 2010, 330, 63.

[17] R. Zacharia, H. Ulbricht, T. Hertel, Phys. Rev. B 2004, 69, 155406.

[18] X. Li, X. Wu, J. Yang, J. Am. Chem. Soc. 2014, 136, 11065.

[19] D. Mukherjee, P. M. Austeria, S. Sampath, ACS Energy Lett. 2016, $1,367$.

[20] J. Zheng, H. Zhang, S. Dong, Y. Liu, C. T. Nai, H. S. Shin, H. Y. Jeong, B. Liu, K. P. Loh, Nat. Commun. 2014, 5, 2995.

[21] V. Nicolosi, M. Chhowalla, M. G. Kanatzidis, M. S. Strano, J. N. Coleman, Science 2013, 340, 1226419.

[22] J. N. Coleman, Acc. Chem. Res. 2012, 46, 14.

[23] Y. Hernandez, V. Nicolosi, M. Lotya, F. M. Blighe, Z. Sun, S. De, I. McGovern, B. Holland, M. Byrne, Y. K. Gun'Ko, Nat. Nanotechnol. 2008, 3, 563.

[24] J. N. Coleman, M. Lotya, A. O'Neill, S. D. Bergin, P. J. King, U. Khan, K. Young, A. Gaucher, S. De, R. J. Smith, Science 2011, 331, 568.

[25] T. Björkman, A. Gulans, A. V. Krasheninnikov, R. M. Nieminen, Phys. Rev. Lett. 2012, 108, 235502.

[26] M. Scagliotti, M. Jouanne, M. Balkanski, G. Ouvrard, G. Benedek, Phys. Rev. B 1987, 35, 7097

[27] K. Z. Du, X. Z. Wang, Y. Liu, P. Hu, M. I. B. Utama, C. K. Gan, Q. Xiong, C. Kloc, ACS Nano 2015, 10, 1738

[28] X. Wang, K. Du, Y. Y. F. Liu, P. Hu, J. Zhang, Q. Zhang, M. H. S. Owen, X. Lu, C. K. Gan, P. Sengupta, 2D Mater. 2016, 3, 031009.

[29] Y. Mathey, R. Clement, C. Sourisseau, G. Lucazeau, Inorg. Chem. 1980, 19, 2773.

[30] Y. C. Zhang, Z. N. Du, K. W. Li, M. Zhang, D. D. Dionysiou, ACS Appl. Mater. Interfaces 2011, 3, 1528.

[31] P. Chen, Y. Su, H. Liu, Y. Wang, ACS Appl. Mater. Interfaces 2013, 5, 12073.

[32] Z. Cheng, F. Wang, T. A. Shifa, C. Jiang, Q. Liu, J. He, Small 2017, 13,1702163 . 